\title{
Singing Mice, Songbirds, and More: Models for FOXP2 Function and Dysfunction in Human Speech and Language
}

\author{
Stephanie A. White, ${ }^{1}$ Simon E. Fisher, ${ }^{3}$ Daniel H. Geschwind, ${ }^{2}$ Constance Scharff, ${ }^{4}$ and Timothy E. Holy ${ }^{5}$ \\ Departments of ${ }^{1}$ Physiological Science and ${ }^{2}$ Neurology, University of California, Los Angeles, California $90095,{ }^{3}$ Wellcome Trust Centre for Human \\ Genetics, University of Oxford, Oxford, United Kingdom, ${ }^{4}$ Max Planck Institute for Molecular Genetics, D-14195 Berlin, Germany, and ${ }^{5}$ Department of \\ Anatomy and Neurobiology, Washington University, St. Louis, Missouri 63110
}

In 2001, a point mutation in the forkhead box P2 (FOXP2) coding sequence was identified as the basis of an inherited speech and language disorder suffered by members of the family known as "KE." This mini-symposium review focuses on recent findings and research-inprogress, primarily from five laboratories. Each aims at capitalizing on the FOXP2 discovery to build a neurobiological bridge between molecule and phenotype. Below, we describe genetic through behavioral techniques used currently to investigate FoxP2 in birds, rodents, and humans for discovery of the neural bases of vocal learning and language.

Key words: basal ganglia; birdsong; brain development; chromatin immunoprecipitation; Forkhead; FOXP2; language; motor learning; song; speech; zebra finch

Language is unique to humans, so how can neuroscientists study its molecular basis? The discovery that mutations in the human gene encoding forkhead box P2 (FOXP2), a transcription factor, result in speech and language deficits (Lai et al., 2001; MacDermot et al., 2005) provides a molecular toehold into exploration of the neural mechanisms for language. However, whose nervous system should be traversed? Humans must be explored, especially for understanding how human brains uniquely recombine a finite set of sounds to generate infinite meaning (Hauser et al., 2002). Another component of language, vocal learning, is also rare but not unique to humans. Vocal learners are animals with a talent for modifying innate vocalizations to imitate or create new sounds. Human speech and birdsong are the best characterized exemplars of vocal learning, and the experimentally tractable songbird has provided molecular and physiological insights. In contrast, transgenically tractable rodents are not thought to learn their vocalizations; however, the recent discovery that male mice produce ultrasonic sounds that are song like (Holy and Guo, 2005) reopens the question of whether such songs are learned.

Given the complexity of language and the variety of speech and language disorders (which affect up to 1 in 20 children), no single tissue or animal model is likely adequate for discovery of the neural bases. Fortunately, a model system need not capture every aspect of a behavior or disorder to be useful. Below, scientists, whose interests range from basic brain-behavior relation-

Received Aug. 4, 2006; revised Aug. 17, 2006; accepted Aug. 21, 2006.

This work was supported by National Institutes of Health (NIH) Grant MH070712 (S.A.W.), The Royal Society, Wellcome Trust, and United Kingdom Medical Research Council (S.E.F.), NIH Grant MH075028 (D.H.G.), The German Science Foundation Sonderforschungsbereich 515, 665 (C.S.), and NIH Grant DC005964 and the Pew Scholars Program (T.E.H.).

Correspondence should be addressed to Stephanie A. White, University of California, Los Angeles, Department of Physiological Science, 621 Charles E. Young Drive South, Los Angeles, CA 90095-1606. E-mail: sawhite@ucla.edu. DOI:10.1523/JNEUROSCI.3379-06.2006

Copyright $\odot 2006$ Society for Neuroscience $\quad 0270-6474 / 06 / 2610376-04 \$ 15.00 / 0$ ships to human cognitive specializations, apply their expertise and model systems to gain understanding of the neural basis of vocal learning and language. Given the firm association between FOXP2 mutations and language deficits, these scientists aim to identify the gene targets of this transcriptional regulator and to investigate the evolutionary, developmental, and real-time roles of FoxP2 in bona fide vocal learners, with a prospective of developing additional models.

Investigating FOXP2 mutations: humans, cell lines, and mutant mice (S. E. Fisher)

Genetic mapping in an unusual multigenerational family exhibiting a monogenic communication disorder (Fisher et al., 1998) provided the first link between FOXP2 and language (Lai et al., 2001). Affected members of the "KE" family carry a heterozygous point mutation, yielding an amino acid substitution $(\mathrm{R} 553 \mathrm{H})$ in the DNA-binding domain of the FOXP2 protein. This tiny change correlates with a multifaceted phenotype (Marcus and Fisher, 2003; Vargha-Khadem et al., 2005) that includes profound deficits in learning and production of complex sequences of mouth movements, impairing speech (verbal dyspraxia), as well as wide-ranging problems with language, extending beyond expressive domains (Watkins et al., 2002a). Despite knowing the primary cause of this disorder, we have little understanding of how FOXP2 exerts its influence(s) on the brain. The Fisher laboratory and collaborators are using three complementary strategies to bridge this gene-brain gap.

\section{Additional studies in humans}

To date, insights into the FOXP2-associated disorder come predominantly from behavioral and neuroimaging studies of a single mutation, $\mathrm{R} 553 \mathrm{H}$, on one background, that of the KE family. These early efforts identified anomalies in subcortical structures (including caudate and cerebellum) (Watkins et al., 2002b) and 
abnormal patterns of cortical activation during language-based tasks (Liegeois et al., 2003). To more fully reveal complex genotype-phenotype relationships, we screened the entire coding region of FOXP2 in 49 probands diagnosed with verbal dyspraxia. We found a novel nonsense mutation (R328X) that truncates the protein and segregates with disorder in relatives of the proband (MacDermot et al., 2005). Detailed phenotypic studies of the family are underway. We and others are also studying cases of chromosomal rearrangement involving the FOXP2 locus. These include translocations (Lai et al., 2001; Shriberg et al., 2006) as well as deletions that involve multiple genes (Liegeois et al., 2001; Zeesman et al., 2006).

\section{Cell lines}

Given the ethical and practical limitations to human studies, analyses of FOXP2 function using human neuron-like cells grown in the laboratory, although potentially "reductionist," can be highly informative. Such systems enabled us to assess disturbances in subcellular localization, DNA binding, and transactivation properties associated with the $\mathrm{R} 553 \mathrm{H}$ and $\mathrm{R} 328 \mathrm{X}$ mutations (Vernes et al., 2006). Moreover, in vitro models can be used to identify other elements in FOXP2-related pathways. For example, the Fisher laboratory and that of D. H. Geschwind have each used chromatin immunoprecipitation to isolate fragments of DNA that are directly bound by FOXP2 protein in living neurons, allowing us to successfully isolate downstream targets (see below, section by D. H. Geschwind).

\section{Mouse models}

Orthologs of FOXP2 are highly conserved across distant vertebrates in both coding sequence and CNS expression (Lai et al., 2003; Haesler et al., 2004; Teramitsu et al., 2004; Bonkowsky and Chien, 2005). Expression patterns suggest that FOXP2 in a common vertebrate ancestor may have influenced the emergence of circuits involved in sensory processing, sensorimotor integration, and control of skilled coordinated movements (Scharff and Haesler, 2005; Fisher and Marcus, 2006). Mice are highly effective systems for genetic manipulation, and we produced an allelic series of mice that carry Foxp 2 point mutations identical to those producing language disorders in humans. These models are important for studying etiological pathways at multiple levels (molecular, cellular, morphological, developmental, electrophysiological, and behavioral). Furthermore, although songbirds presently represent the model of choice for uncovering mechanisms involved in vocal learning (see below, sections by S. A. White and C. Scharff), mice still offer perspectives about the roles of genes in vocalizations. For example, a recent study by another group (Shu et al., 2005) reported that ablation of Foxp2 in their targeted knock-out correlated with a reduction in the number of isolation calls made by mouse pups when removed from their mother, although no abnormalities in call structure were noted. Accordingly, we are in the midst of performing detailed analyses of the vocalizations in our Foxp2 mutant lines. Intriguingly, Holy and Guo (2005) have discovered that vocalizations of adult male mice are more complex than previously appreciated and share characteristics of birdsong (see below, section by T. E. Holy). Whether or not such vocalizations are learned, it will be interesting to determine whether/how Foxp2 mutations affect the properties of these "mouse songs."

\section{Investigating FOXP2 function in cognition: genomic screening for FOXP2 targets in humans (D. H. Geschwind)} Evidence from many sources demonstrates a strong but complex genetic component for language that consists of many loci and interacts with environmental factors (Fisher et al., 2003). Although rare, monogenic disorders of speech and language, such as those caused by FOXP2 mutation, provide a unique window through which to study the biological basis of speech and language in health and disease. Thus, one of the goals of the Geschwind laboratory is to identify downstream targets of FOXP2 that may be relevant to human brain development, so as to learn more about the molecular events driving this complex process. Development of regional identity in the mammalian telencephalon occurs over a protracted period and is modifiable until the middle to late stages of neurogenesis and migration (in humans, 10-25 weeks gestation) (Barbe and Levitt, 1995; McConnell, 1995; Rakic, 1995; Fishell, 1997; Nothias et al., 1998; Rakic and Lombroso, 1998; Rubenstein and Beachy, 1998). This is thus a critical time to identify and study gene products involved in the development of human higher functions such as speech and language (Geschwind and Miller, 2001; Geschwind et al., 2002; Sun et al., 2005), including the effects of FOXP2 on the basal ganglia and cortex.

The Geschwind laboratory has developed a staged genomic screening approach using chromatin immunoprecipitation coupled to microarray analysis (ChIP-Chip) to identify potential neural targets of human FOXP2, both in vitro and in vivo. ChIPChip enables the genome-wide study of direct interactions between a protein and the chromosomal sites to which it binds, in the context of the normal chromatin structure of living tissue (Ren et al., 2000; Ren and Dynlacht, 2004). Briefly, cells or tissues are treated with a crosslinking agent, and then an antibody recognizing the protein of interest is used to selectively immunoprecipitate protein-DNA complexes. After reversing crosslinks, the recovered DNA is hybridized to arrays containing DNA from thousands of human genes, allowing systematic identification of transcription factor binding sites. For these experiments, we made high-affinity, specific polyclonal antibodies based on unique C-terminal regions of FOXP2. In vitro, ChIP exploited SH-SY5Y human neuroblastoma cells, after induction of FOXP2 expression via $\mathrm{BDNF} /$ retinoic acid treatment, whereas in vivo, ChIP involved material from human basal ganglia and inferior frontal cortex at midgestation. In each case, experiments were performed in triplicate, and the DNA that was pulled down was hybridized to microarrays containing $\sim 700 \mathrm{bp}$ of the promoter regions and $300 \mathrm{bp}$ from the intronic regions of $\sim 6000$ human genes (Aviva Systems Biology Corporation, San Diego, CA). Target sequences are found in genes from a variety of gene ontology categories, including those involved in neural development.

ChIP-Chip can identify regions of transcription factor binding at high specificity (Ren et al., 2000; Ren and Dynlacht, 2004; Kim et al., 2005). However, it does not determine whether binding has a functional effect on the putative target gene or whether binding results in transcriptional repression or activation. In the last stage of this genomic screening experiment, we used small interfering RNA and overexpression in vitro to demonstrate functional effects of FOXP2 binding on target genes suggested by ChIP-Chip. The function and expression of these genes vis-à-vis the development of circuits involved in speech and language and disorders disrupting them (e.g., autism; specific language impairment) are now important avenues of our research.

\section{Dynamic regulation of FoxP2 during singing (S. A. White)}

The corticostriatal abnormalities that accompany verbal dyspraxia in humans bearing FOXP2 mutations implicate FOXP2 in the ontogenesis of neural circuitry involved in speech and language. Accordingly, the powerful strategies outlined above use 
FOXP2 as a molecular entry point toward understanding how the brain develops this capacity. The White laboratory and that of C. Scharff (see below, section by C. Scharff) have used songbirds as behaviorally relevant and physiologically accessible models to determine whether FOXP2 additionally functions during vocal learning and in adulthood (Scharff and White, 2004). To address the question of a real-time role during vocalization, the White laboratory has examined FoxP2 mRNA expression in zebra finches, a songbird species in which males sing stable, unchanging songs to court females. Any alteration in FoxP2 within the song control regions of adult singers would thus reflect real-time changes rather than the developmental or seasonal ones, discussed below.

Male zebra finches offer another potential insight to a behavioral role of FoxP2 because they display two basic types of singing: "directed" singing is when a male performs to a female; "undirected" singing is when a male practices alone or sings in the presence of, but not toward, conspecifics (Zann, 1996). These acoustically similar yet socially distinct vocal behaviors allowed us to address whether any mature function of FoxP2 is purely motor or also contingent on social context (Jarvis et al., 1998).

We found that FoxP2 mRNA declines rapidly and specifically within the striatal song control region Area $\mathrm{X}$ when males sing but is stable in nonsinging birds. Furthermore, this decline occurs when males practice alone but not when they perform to females (Teramitsu and White, 2006). This real-time regulation of FoxP2 during vocalization, dependent on social context, suggests that FoxP2 functions beyond development and beyond pure motor control. Obtained from a humble grass finch, these data nonetheless support the conclusion that human FOXP2 mutations entail more than motor deficits (Watkins et al., 2002a) and suggest, by analogy, postdevelopmental roles for FOXP2 in human speech.

\section{FoxP2 in brain evolution, development, and vocal learning: more perspectives from the birds (C. Scharff)}

FOXP2 has undergone recent positive selection in human history (Enard et al., 2002; Zhang et al., 2002). Given the established finding that FOXP2 mutations lead to speech and language disorders, it is tempting to speculate that the adaptive evolution of human FOXP2 may have related to the emergence of modern speech capacities (Enard et al., 2002; Zhang et al., 2002). Among birds, however, there is no evidence that Darwinian selection of changes in the FoxP2 protein sequence contributed to vocal learning in some, but not other, avian species (Webb and Zhang, 2005). Given this evolutionary difference, can findings from songbirds really be relevant to the role of FOXP2 in human speech? Perhaps. To begin, despite some differences, the FoxP2 protein of songbirds is astonishingly similar to mammalian FoxP2: for example, the zebra finch and human sequences share $100 \%$ identity within the DNA-binding domain (Haesler et al., 2004; Teramitsu et al., 2004). A strong conservation like this often indicates shared function. Furthermore, the FoxP2 expression pattern in the brain of many birds that learn their songs by imitation is very similar to that of rodents and humans, including expression within the same cell types, such as striatal medium spiny neurons (Scharff and Haesler, 2005). FoxP2 is expressed early in the embryo and remains "on" in some regions into adulthood. What does this have to do with speech? It suggests that FoxP2 is necessary to allow brain regions involved in vocal behavior to develop properly during embryonic life (see above, sections by S. E. Fisher and D. H. Geschwind). If so, does it fulfill a related, or a different, role later in life, after the brain has finished its "construction phase"?
To address the latter question, the Scharff laboratory examined FoxP2 mRNA expression in young male zebra finches. We found that, when the birds are in full swing of learning to sing, there is more FoxP2, bilaterally in Area X (Haesler et al., 2004), the striatal region known to be vital for song learning (Sohrabji et al., 1990; Scharff and Nottebohm, 1991). This finding suggests that FoxP2 might be directly involved in the learning, but perhaps this is just coincidence. However, adult canaries also have higher FoxP2 expression in Area X exactly during those months of the year when they remodel their song after having previously sung very stable song to woo females (Haesler et al., 2004). Coincidence again? To find out whether FoxP2 is required for song behavior, we need to get rid of it, at the right time, in the right place. Because knock-out technology is not yet available for songbirds, we use RNA interference to downregulate FoxP2 in Area X. These studies, still ongoing, should soon provide answers to the following questions. Can zebra finches still sing normal song after growing up with less FoxP2 in Area X than normally present? Even more interestingly, will they still copy the song of an adult male tutor? By selecting an animal model (here, a songbird) that exhibits key aspects of the behaviors affected in humans with FOXP2 mutations (here, vocal learning), the potential for observing the effects of functional intervention can be realized.

\section{Analyzing the ultrasonic songs of male mice (T. E. Holy)}

Although the most widely appreciated vocalizations of mice are audible, it has been known for several decades that mice and other rodents also vocalize at ultrasonic frequencies. In the Holy laboratory, we recorded and analyzed the vocalizations of a sizable population of adult males. When the recordings were computationally shifted into the range of human hearing, these vocalizations were found to be subjectively reminiscent of bird songs.

To objectively determine the extent of similarity with bird songs, individual syllables were analyzed quantitatively (Holy and Guo, 2005). Mouse syllables can be classified into distinct types, with several different approaches yielding consistent classifications. These vocalizations are also structured in time. During a bout of vocalization, syllable types are arranged in nonrandom order, with the most obvious characteristic being that particular types tend to be repeated several times before a new syllable type is uttered. Finally, individual mice show reproducible biases in terms of their syllable type usage and degree of repetitiveness, traits that reliably distinguish them from other males. Consequently, these vocalizations have many of the characteristics that have been described for birdsong.

Recently, we further examined the stimulus triggers for these songs. Previous work has shown that males vocalize in the presence of females and also when they encounter mouse pheromones, chemosensory cues detected by the olfactory system. We examined whether the songs uttered in the presence of male cues are different from those triggered by female cues. We find that the songs differ greatly in terms of degree (with far more singing triggered by female than by male cues) but do not find any consistent difference in the character of the songs. Together, these discoveries increase the attractiveness of mice as model systems for study of vocalizations (see above, section by S. E. Fisher).

\section{Conclusion}

The first report of FOXP2 as a monogenetic locus of a speech and language disorder has enabled great strides in the design and execution of experiments that bring us closer to understanding the biological basis of vocal learning and language. These reflect 
the creativity and power of comparative and integrative approaches for understanding behavior, including those that are cognitively complex.

\section{References}

Barbe MF, Levitt P (1995) Age-dependent specification of the corticocortical connections of cerebral grafts. J Neurosci 15:1819-1834.

Bonkowsky JL, Chien CB (2005) Molecular cloning and developmental expression of foxP2 in zebrafish. Dev Dyn 234:740-746.

Enard W, Przeworski M, Fisher SE, Lai CSL, Wiebe V, Kitano T, Monaco AP, Paeaebo S (2002) Molecular evolution of FOXP2, a gene involved in speech and language. Nature 418:869-872.

Fishell G (1997) Regionalization in the mammalian telencephalon. Curr Opin Neurobiol 7:62-69.

Fisher SE, Marcus GF (2006) The eloquent ape: genes, brains and the evolution of language. Nat Rev Genet 7:9-20.

Fisher SE, Vargha-Khadem F, Watkins KE, Monaco AP, Pembrey ME (1998) Localisation of a gene implicated in a severe speech and language disorder. Nat Genet 18:168-170.

Fisher SE, Lai CS, Monaco AP (2003) Deciphering the genetic basis of speech and language disorders. Annu Rev Neurosci 26:57-80.

Geschwind DH, Miller BL (2001) Molecular approaches to cerebral laterality: development and neurodegeneration. Am J Med Genet 101:370-381.

Geschwind DH, Miller BL, DeCarli C, Carmelli D (2002) Heritability of lobar brain volumes in twins supports genetic models of cerebral laterality and handedness. Proc Natl Acad Sci USA 99:3176-3181.

Haesler S, Wada K, Nshdejan A, Morrisey EE, Lints T, Jarvis ED, Scharff C (2004) FoxP2 expression in avian vocal learners and non-learners. J Neurosci 24:3164-3175.

Hauser MD, Chomsky N, Fitch WT (2002) The faculty of language: what is it, who has it, and how did it evolve? Science 298:1569-1579.

Holy TE, Guo Z (2005) Ultrasonic songs of male mice. PLoS Biol 3:e386.

Jarvis ED, Scharff C, Grossman MR, Ramos JA, Nottebohm F (1998) For whom the bird sings: context-dependent gene expression. Neuron 21:775-788.

Kim TH, Barrera LO, Zheng M, Qu C, Singer MA, Richmond TA, Wu Y, Green RD, Ren B (2005) A high-resolution map of active promoters in the human genome. Nature 436:876-880.

Lai CS, Fisher SE, Hurst JA, Vargha-Khadem F, Monaco AP (2001) A forkhead-domain gene is mutated in a severe speech and language disorder. Nature 413:519-523.

Lai CS, Gerrelli D, Monaco AP, Fisher SE, Copp AJ (2003) FOXP2 expression during brain development coincides with adult sites of pathology in a severe speech and language disorder. Brain 126:2455-2462.

Liegeois FJ, Lai CS, Baldeweg T, Fisher SE, Monaco AP, Connelly A, VarghaKhadem F (2001) Behavioural and neuroimaging correlates of a chromosome $7 \mathrm{q} 31$ deletion containing the SPCH1 gene. Soc Neurosci Abstr 27:529.17.

Liegeois FJ, Baldeweg T, Connelly A, Gadian DG, Mishkin M, VarghaKhadem F (2003) Language fMRI abnormalities associated with FOXP2 gene mutation. Nat Neurosci 6:1230-1237.

MacDermot KD, Bonora E, Sykes N, Coupe AM, Lai CS, Vernes SC, VarghaKhadem F, McKenzie F, Smith RL, Monaco AP, Fisher SE (2005) Identification of FOXP2 truncation as a novel cause of developmental speech and language deficits. Am J Hum Genet 76:1074-1080.

Marcus GF, Fisher SE (2003) FOXP2 in focus: what can genes tell us about speech and language? Trends Cogn Sci 7:257-262.

McConnell SK (1995) Plasticity and commitment in the developing cerebral cortex. Prog Brain Res 105:129-143.

Nothias F, Fishell G, Ruiz i Altaba A (1998) Cooperation of intrinsic and extrinsic signals in the elaboration of regional identity in the posterior cerebral cortex. Curr Biol 8:459-462.
Rakic P (1995) Radial versus tangential migration of neuronal clones in the developing cerebral cortex. Proc Natl Acad Sci USA 92:11323-11327.

Rakic P, Lombroso PJ (1998) Development of the cerebral cortex. I. Forming the cortical structure. J Am Acad Child Adolesc Psychiatry $37: 116-117$.

Ren B, Dynlacht BD (2004) Use of chromatin immunoprecipitation assays in genome-wide location analysis of mammalian transcription factors. Methods Enzymol 376:304-315.

Ren B, Robert F, Wyrick JJ, Aparicio O, Jennings EG, Simon I, Zeitlinger J, Schreiber J, Hannett N, Kanin E, Volkert TL, Wilson CJ, Bell SP, Young RA (2000) Genome-wide location and function of DNA binding proteins. Science 290:2306-2309.

Rubenstein JL, Beachy PA (1998) Patterning of the embryonic forebrain. Curr Opin Neurobiol 8:18-26.

Scharff C, Haesler S (2005) An evolutionary perspective on FoxP2: strictly for the birds? Curr Opin Neurobiol 15:694-703.

Scharff C, Nottebohm F (1991) A comparative study of the behavioural deficits following lesions of various parts of the zebra finch song system: implications for vocal learning. J Neurosci 19:2896-2913.

Scharff C, White SA (2004) Genetic components of vocal learning. Ann NY Acad Sci 1016:325-347.

Shriberg LD, Ballard KJ, Tomblin JB, Duffy JR, Odell KH (2006) Speech, prosody, and voice characteristics of a mother and daughter with a 7;13 translocation affecting FOXP2. J Speech Lang Hear Res 49:500-525.

Shu W, Cho JY, Jiang Y, Zhang M, Weisz D, Elder GA, Schmeidler J, De Gasperi R, Sosa MA, Rabidou D, Santucci AC, Perl D, Morrisey E, Buxbaum JD (2005) Altered ultrasonic vocalization in mice with a disruption in the Foxp2 gene. Proc Natl Acad Sci USA 102:9643-9648.

Sohrabji F, Nordeen EJ, Nordeen KW (1990) Selective impairment of song learning following lesions of a forebrain nucleus in the juvenile zebra finch. Behav Neur Biol 53:51-63.

Sun T, Patoine C, Abu-Khalil A, Visvader J, Sum E, Cherry JT, Orkin SH, Geschwind DH, Walsh CA (2005) Early asymmetry of gene transcription in embryonic human left and right cerebral cortex. Science 308:1794-1798.

Teramitsu I, White SA (2006) FoxP2 regulation during undirected singing in adult songbirds. J Neurosci 26:7390-7394.

Teramitsu I, Kudo LC, London SE, Geschwind DH, White SA (2004) Parallel FoxP1 and FoxP2 expression in songbird and human brain predicts functional interaction. J Neurosci 24:3152-3163.

Vargha-Khadem F, Gadian DG, Copp A, Mishkin M (2005) FOXP2 and the neuroanatomy of speech and language. Nat Rev Neurosci 6:131-138.

Vernes SC, Nicod J, Elahi FM, Coventry JA, Kenny N, Coupe A-M, Bird LE, Davies KE, Fisher SE (2006) Functional genetic analysis of mutations implicated in a human speech and language disorder. Hum Mol Genet, in press.

Watkins KE, Dronkers NF, Vargha-Khadem F (2002a) Behavioural analysis of an inherited speech and language disorder: comparison with acquired aphasia. Brain 125:452-464.

Watkins KE, Vargha-Khadem F, Ashburner J, Passingham RE, Connelly A, Friston KJ, Frackowiak RS, Mishkin M, Gadian DG (2002b) MRI analysis of an inherited speech and language disorder: structural brain abnormalities. Brain 125:465-478.

Webb DM, Zhang J (2005) FoxP2 in song-learning birds and vocal-learning mammals. J Hered 96:212-216.

Zann RA (1996) The zebra finch: a synthesis of field and laboratory studies, pp 157-246. New York: Oxford UP.

Zeesman S, Nowaczyk MJ, Teshima I, Roberts W, Cardy JO, Brian J, Senman L, Feuk L, Osborne LR, Scherer SW (2006) Speech and language impairment and oromotor dyspraxia due to deletion of $7 \mathrm{q} 31$ that involves FOXP2. Am J Med Genet A 140:509-514.

Zhang J, Webb DM, Podlaha O (2002) Accelerated protein evolution and origins of human-specific features: Foxp2 as an example. Genetics 162: 1825-1835. 